\title{
Sodium Fluoride Positron Emission Tomography/Computed Tomography of the Whole Body
}

National Cancer Institute

\section{Source}

National Cancer Institute. Sodium Fluoride Positron Emission Tomography/Computed

Tomography of the Whole Body. NCI Thesaurus. Code C137906.

Positron emission tomography combined with computed tomography of the whole body with the use of the contrast agent sodium fluoride F 18 (18F-NaF) to enhance the image. 